\title{
Diseño y Validación de un Ciclón Stairmand Destinado al Filtrado Parcial de Material Particulado
}

\author{
Design and Validation of a Stairmand Cyclone for Partial Filtering of Particulate \\ Material
}

Eliel Eduardo Montijo-Valenzuela

\section{ఠ \\ EDICIÓN: \#-CIVTAC}

Recibido: 28/diciembre/2020

Aceptado: 28/marzo/2021

Publicado: 16/abril/2021

País

${ }^{1}$ México

\section{IIII Institución}

${ }^{1}$ Universidad Internacional Iberoamericana (UNINI)

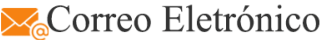 \\ 1elielmontijo@gmail.com}

\section{(iD) ORCID}

'https://orcid.org/0000-0001-8538-0767

\section{Citar así: CfAPA / IEEE}

Montijo-Valenzuela, E. (2021). Diseño y validación de un ciclón Stairmand destinado al filtrado parcial de material particulado. Revista TecnológicaEducativa Docentes 2.0, 11(1), 80-88. https://doi.org/10.37843/rted.v11i1.196

E. Montijo-Valenzuela, "Diseño y validación de un ciclón Stairmand destinado al filtrado parcial de material particulado", RTED, vol. 11, n. ${ }^{\circ}$, pp. 8088, abr. 2021.

\section{Resumen}

Los materiales particulados incluyen solidos o líquidos o una mezcla de ellos a escalas micrométricas. Estas partículas, pueden ser perjudiciales para el medio ambiente y se asocia a algunos problemas de salud humana, debido principalmente a su tamaño. Por esta situación se han creado tecnologías para su mitigación, como los ciclones. Esta tecnología aprovecha la energía cinética del movimiento de flujo contaminado para efectuar una separación de forma mecánica, por ello son implementados en diversas aplicaciones industriales como en los prelimpiadores de líquidos o gases. El objetivo de esta investigación fue diseñar un ciclón de alta eficiencia tipo Stairmand para separación de partículas sólidas en un flujo gaseoso, con una eficiencia del $80 \%$, a partir de un problema de estudio, donde se conocen los parámetros iniciales junto a las variables implicadas en el sistema. El proceso metodológico empleado para esta investigación se centró en un estudio descriptivo-correlacional, siguiendo los siguientes pasos: 1) clasificación de las variables para calculo teórico, diseño tridimensional con análisis de simulación, 2) determinación de los parámetros geométricos teóricos, 3) diseño y modelado de ciclón en software Solidworks ${ }^{\circledR}$, 4) cálculo teórico de eficiencia de colección, 5) análisis de eficiencia de colección con Solidworks® Flow Simulation a partir de resultados de simulación. Los resultados teóricos, aunados a los de simulación mostraron una coincidencia con error inferior a 1\%, demostrando la hipótesis planteada en esta investigación.

Palabras clave: Diseño, validación, ciclón stairmand, filtrado parcial.

\section{Abstract}

Particulate materials include solids or liquids or a mixture of them at micrometer scales. These particles can be harmful to the environment and are associated with some human health problems, mainly due to their size. Because of this situation, technologies have been created for their mitigation, such as cyclones. This technology takes advantage of the kinetic energy of the contaminated flow movement to perform a mechanical separation, so they are implemented in various industrial applications such as pre-cleaners of liquids or gases. This research was aimed to design a high-efficiency Stairmand type cyclone for the separation of solid particles in a gaseous flow, with an efficiency of $80 \%$, based on a study problem, where the initial parameters are known together with the variables involved in the system. The methodological process employed for this research focused on a descriptivecorrelational study, following the following steps: 1) classification of variables for theoretical calculation, three-dimensional design with simulation analysis, 2) determination of theoretical geometric parameters, 3) cyclone design and modeling in Solidworks ${ }^{\circledR}$ software, 4) theoretical calculation of collection efficiency, 5) collection efficiency analysis with Solidworks ${ }^{\circledR}$ Flow Simulation from simulation results. Together with the simulation results. The theoretical results showed a coincidence with an error of less than $1 \%$, demonstrating the hypothesis put forward in this research.

Keywords: Vermicompost, organic waste, interactive and integrating. 


\section{Introducción}

El material particulado (PM) es un término empleado para describir una mezcla de partículas en estado sólido o gotas líquidas presentes en el aire (EPA, 2020). La generación del PM se debe a fuentes de contaminación naturales o de tipo antropogénicas, como consecuencia de actividades del ser humano (Echeverri, 2019), teniendo serias implicaciones en suelo, aire y agua (Sotomayor, 2018), incluso en la salud humana; principalmente relacionada con problemas cardiovasculares (Khaniabadi et al., 2016; Mannucci, 2017; Fiordelisi et al., 2017; An et al., 2018; Kirrane et al., 2019; Yin et al., 2020; Hadei \& Naddafi, 2020), sumados a los respiratorios (Yao et al., 2018; Sicard et al., 2019; Leikauf, Kim \& Jang, 2020; Kyung \& Jeong; 2020).

Como medida precautoria para mitigar las emisiones antropogénicas de PM, se han implementado diversas estrategias (Sofia, 2020), tecnologías domésticas (World Health Organization, 2020), tecnologías industriales (Kwiatkowski et al., 2019), entre estas últimas, los ciclones. Los separadores ciclónicos son reconocidos y aceptados en diversas aplicaciones, generalmente del tipo industrial, entre ellas, el condensado de vapor, colección de polvos en hornos (Wang et al., 2019; Cao \& Bian, 2019; Wasilewski \& Brar, 2019), o en elaboración de alimentos, minería y construcción (Gamiño et al., 2018; EPA-CICA, 2012). Los ciclones de alta eficiencia Stairmand, manejan una separación eficiente de MP en diámetros aerodinámicos de entre 5 a $10 \mu \mathrm{m}$ (Gamiño et al., 2018), con una eficiencia de colección promedio del $80 \%$.

Debido al complejo nivel de operación y las variables de entrada-salida, el rendimiento de los diseños de ciclones es validado mediante simulación fluidodinámica (Makwana \& Lakdawala, 2016; Vakamalla et al., 2016; Kumar \& Jha, 2018; Gopalakrishnan \& Arul-Prakash, 2019). La simulación fluidodinámica o dinámica de fluidos computacionales (CFD, por sus siglas en inglés), es una herramienta para resolver numéricamente las ecuaciones del movimiento de los fluidos con uso de computadora (Xamán \& Girón, 2015). En trabajo recientes, el uso de herramientas CFD es aplicado a ciclones para análisis de optimización de modelos, incluyendo cambios en geometrías (Luciano et al., 2018), en este contexto, existen investigaciones dentro de literatura, que centran métodos de validación de eficiencia en separación con ciclones, a partir de los cálculos teóricos en conjunto con CFD.

Con base a lo anterior, en esta investigación se toma como referencia el siguiente caso de estudio: Diseñar un ciclón para separar sólidos de una corriente gaseosa. La densidad de las partículas es de $1500 \mathrm{~kg} / \mathrm{m}^{3}$ y el gas es aire a $450{ }^{\circ} \mathrm{C}$. El caudal de la corriente es $3.2 \mathrm{~m}^{3} / \mathrm{s}$, y la operación es a una presión de 85.3 $\mathrm{kPa}$. La concentración de las partículas es de 2.0 $\mathrm{g} / \mathrm{m}^{3} \mathrm{y}$, según las normas de emisión, se requiere una eficiencia de separación del 80\% (Echeverri, 2006, p. 135). El objetivo general de esta investigación fue diseñar un ciclón de alta eficiencia tipo Stairmand utilizando los parámetros establecidos en (Echeverri, 2006) junto con el software Solidworks ${ }^{\circledR}$, para posteriormente validar el porcentaje de eficiencia de colección utilizando los análisis de Solidworks ${ }^{\circledR}$ Flow Simulation.

\section{Metodología}

Esta investigación es de carácter cuantitativa cuyo alcance se basa en la revisión de literatura y estudios relacionados al campo de aplicación, específicamente de carácter descriptivo y correlacional. El primero busca una especificación de propiedades, sumado a una consideración del fenómeno de estudio y sus componentes, para medir conceptos y definir variables. El segundo permite una asociación entre los conceptos y variables, permitiendo predicciones y cuantificaciones (HernándezSampieri \& Mendoza-Torres, 2014).

El alcance de esta investigación está orientado a un estudio descriptivo-correlacional. El estudio descriptivo se asocia a las especificaciones de carácter geométricas y de diseño, utilizando metodologías teóricas, aunadas a las de simulación. En el estudio correlacional, se pretende medir el grado de eficiencia de los cálculos teóricos, junto a ecuaciones aplicables con los valores obtenidos por CFD. 
Diseño y validación de un ciclón Stairmand destinado al

Tabla 1

Clasificación de las variables implicadas en el cálculo teórico y diseño del ciclón tipo Stairmand

\begin{tabular}{|c|c|c|c|c|c|}
\hline \multirow{2}{*}{$\begin{array}{l}\text { Nombre } \\
\text { de la variable }\end{array}$} & \multirow[t]{2}{*}{ Representación } & \multicolumn{2}{|c|}{ Tipo } & \multicolumn{2}{|c|}{ Naturaleza } \\
\hline & & Independiente & Dependiente & Cuantitativa & Cualitativa \\
\hline $\begin{array}{l}\text { Densidad del } \\
\text { material particulado }\end{array}$ & $\rho$ & $\mathrm{X}$ & & $\mathrm{X}$ & \\
\hline $\begin{array}{l}\text { Temperatura de } \\
\text { operación de la } \\
\text { mezcla de aire y } \\
\text { material particulado }\end{array}$ & $\mathrm{T}$ & $\mathrm{X}$ & & $\mathrm{X}$ & \\
\hline $\begin{array}{l}\text { Caudal de entrada al } \\
\text { sistema }\end{array}$ & Qi & $\mathrm{X}$ & & $\mathrm{X}$ & \\
\hline $\begin{array}{l}\text { Velocidad de entrada } \\
\text { al sistema }\end{array}$ & $\mathrm{Vi}$ & $\mathrm{X}$ & & $\mathrm{X}$ & \\
\hline Presión de operación & Po & & $\mathrm{X}$ & $\mathrm{X}$ & \\
\hline $\begin{array}{l}\text { Concentración de } \\
\text { partículas }\end{array}$ & PM & & $\mathrm{X}$ & $\mathrm{X}$ & \\
\hline Área & A & $\mathrm{X}$ & & $X$ & \\
\hline $\begin{array}{l}\text { Altura de entrada del } \\
\text { ciclón }\end{array}$ & $\mathrm{a}$ & $X$ & & $\mathrm{X}$ & \\
\hline $\begin{array}{l}\text { Ancho de la entrada } \\
\text { del ciclón }\end{array}$ & $\mathrm{b}$ & $\mathrm{X}$ & & $\mathrm{X}$ & \\
\hline $\begin{array}{l}\text { Altura de salida del } \\
\text { ciclón }\end{array}$ & SA & $\mathrm{X}$ & & $\mathrm{X}$ & \\
\hline $\begin{array}{l}\text { Diámetro de salida } \\
\text { del ciclón }\end{array}$ & Ds & $X$ & & $\mathrm{X}$ & \\
\hline $\begin{array}{l}\text { Altura de la parte } \\
\text { cilíndrica del ciclón }\end{array}$ & $\mathrm{h}$ & $\mathrm{X}$ & & $\mathrm{X}$ & \\
\hline $\begin{array}{l}\text { Altura total del } \\
\text { ciclón }\end{array}$ & $\mathrm{H}$ & $X$ & & $X$ & \\
\hline $\begin{array}{l}\text { Altura de la parte } \\
\text { cónica del ciclón }\end{array}$ & Z & $\mathrm{X}$ & & $\mathrm{X}$ & \\
\hline $\begin{array}{l}\text { Diámetro de salida } \\
\text { del polvo }\end{array}$ & B & $X$ & & $X$ & \\
\hline $\begin{array}{l}\text { Velocidad } \\
\text { equivalente }\end{array}$ & Veq & & $X$ & $X$ & \\
\hline $\begin{array}{l}\text { Velocidad } \\
\text { saturación }\end{array}$ & Vsat & & $X$ & $\mathrm{X}$ & \\
\hline Tiempo de retención & $\operatorname{Tr}$ & & $X$ & $X$ & \\
\hline $\begin{array}{l}\text { Eficiencia } \\
\text { colección }\end{array}$ & ni & & $\mathrm{X}$ & $\mathrm{X}$ & \\
\hline Densidad del aire & $\rho g$ & & $X$ & $\mathrm{X}$ & \\
\hline $\begin{array}{l}\begin{array}{l}\text { Densidad de la } \\
\text { partícula }\end{array} \\
\text { le }\end{array}$ & $\rho p$ & $\mathrm{X}$ & & $X$ & \\
\hline $\begin{array}{l}\text { Viscosidad dinámica } \\
\text { del aire }\end{array}$ & $\mu_{\mathrm{A}}$ & & $X$ & $X$ & \\
\hline Vórtice & VRX & & $\mathrm{X}$ & & $\mathrm{X}$ \\
\hline Gravedad & $\mathrm{Gr}$ & $X$ & & $\mathrm{X}$ & \\
\hline $\begin{array}{lll}\begin{array}{l}\text { Diámetro de } \\
\text { partículas }\end{array} & & \text { las } \\
\end{array}$ & Dpi & $\mathrm{X}$ & & $\mathrm{X}$ & \\
\hline $\begin{array}{l}\text { Factor } \\
\text { configuración }\end{array}$ & $\mathrm{Gr}$ & $\mathrm{X}$ & & X & \\
\hline $\begin{array}{l}\text { Número de cabezas } \\
\text { de velocidad }\end{array}$ & $\mathrm{NH}$ & $X$ & & $\mathrm{X}$ & \\
\hline Número de vórtices & $\mathrm{N}$ & $X$ & & & $X$ \\
\hline
\end{tabular}

Nota. Tipos de variables y su naturaleza, implicadas en el cálculo y diseño, elaborado por: Echeverri (2006), Hoffmann et al., (2008), Petit \& Barbosa (2013) y Petit et al., (2012).

La hipótesis de esta investigación se centra en la validación de un ciclón Stairmand y se formula a continuación: se puede validar un modelo teórico de ciclón tipo Stairmand a partir de simulación CFD, implementando los mismos parámetros de entrada tanto en el modelo teórico y el modelo simulado, obteniendo un error no mayor a un 5\% en el total de colección de PM. Las variables se clasifican a través de tablas (ver Tabla 1 y Tabla 2), donde se concentran los aspectos más relevantes en el cálculo teórico y el diseño del ciclón tipo Stairmand, junto con las implicaciones dependientes e independientes en el análisis de CFD, clasificadas por tipo y por naturaleza. Para la tipología se establecen las variables como dependientes e independientes, y para la naturaleza, como cuantitativas y cualitativas. 
Diseño y validación de un ciclón Stairmand destinado al filtrado parcial de material particulado

Tabla 2

Clasificación de las variables implicadas en CFD del ciclón tipo Stairmand

\begin{tabular}{|c|c|c|c|c|c|}
\hline \multirow{2}{*}{$\begin{array}{c}\text { Nombre } \\
\text { de la } \\
\text { variable }\end{array}$} & \multirow{2}{*}{$\begin{array}{c}\text { Rep } \\
\text { re- } \\
\text { sent } \\
\text { ació } \\
\text { n }\end{array}$} & \multicolumn{2}{|c|}{ Tipo } & \multicolumn{2}{|c|}{ Naturaleza } \\
\hline & & $\begin{array}{c}\text { Indepe } \\
\text { ndient } \\
\text { e }\end{array}$ & $\begin{array}{l}\text { Depen } \\
\text { diente }\end{array}$ & $\begin{array}{l}\text { Cuant } \\
\text { itativa }\end{array}$ & $\begin{array}{c}\text { Cual } \\
\text { itativ } \\
\text { a }\end{array}$ \\
\hline $\begin{array}{l}\text { Temperatur } \\
\text { a de } \\
\text { operación } \\
\text { de la mezcla } \\
\text { de aire y } \\
\text { material } \\
\text { particulado }\end{array}$ & $\mathrm{T}$ & & $\mathrm{X}$ & $\mathrm{X}$ & \\
\hline $\begin{array}{l}\text { Caudal de } \\
\text { entrada al } \\
\text { sistema }\end{array}$ & Qi & & $X$ & $\mathrm{X}$ & \\
\hline $\begin{array}{l}\text { Velocidad } \\
\text { de entrada } \\
\text { al sistema }\end{array}$ & Vi & & $\mathrm{X}$ & $\mathrm{X}$ & \\
\hline $\begin{array}{l}\text { Presión de } \\
\text { operación }\end{array}$ & Po & & $X$ & $\mathrm{X}$ & \\
\hline $\begin{array}{l}\text { Densidad } \\
\text { del aire }\end{array}$ & $\rho g$ & & $\mathrm{X}$ & $\mathrm{X}$ & \\
\hline $\begin{array}{l}\text { Densidad de } \\
\text { la partícula }\end{array}$ & $\rho p$ & $\mathrm{X}$ & & $\mathrm{X}$ & \\
\hline $\begin{array}{l}\text { Viscosidad } \\
\text { dinámica } \\
\text { del aire }\end{array}$ & $\mu_{\mathrm{A}}$ & & $\mathrm{X}$ & X & \\
\hline Vórtice & $\begin{array}{l}\text { VR } \\
\mathrm{X}\end{array}$ & & $X$ & & $X$ \\
\hline
\end{tabular}

Nota. Tipos de variables y su naturaleza, implicadas en simulación, elaborado por: Ast Ingeniería (2016), Bahamón et al., (2009), Capote et al., (2008), Gamiño et al., (2018) y Witt et al., (1999).

\section{Ecuaciones para parámetros geométricos de diseño en ciclón Stairmand}

Para el dimensionamiento del ciclón Stairmand, se establecen una serie de ecuaciones determinantes para su diseño, siguiendo el proceso metodológico empleado en (Maduabuchi \& Kuye, 2017; Miller, 2017; Echeverri, 2006), cuyas variables se encuentran caracterizadas en Tablas 1 y 2 , esquematizadas en Figura 1. Las ecuaciones se muestran a continuación (Ecuaciones 1 a 8):

$$
\begin{aligned}
& A=a * b=\frac{Q_{i}}{V_{i}} \\
& a=0.5 \mathrm{Dc} \\
& b=0.2 \mathrm{Dc} \\
& \mathrm{S}=0.5 \mathrm{Dc} \\
& \mathrm{Ds}=0.5 \mathrm{Dc}
\end{aligned}
$$

$$
\begin{aligned}
& \mathrm{h}=1.5 \mathrm{Dc} \\
& \mathrm{z}=2.5 \mathrm{Dc} \\
& \mathrm{B}=0.375 \mathrm{Dc}
\end{aligned}
$$

\section{Figura 1}

Parámetros dimensionales de un ciclón tipo Stairmand

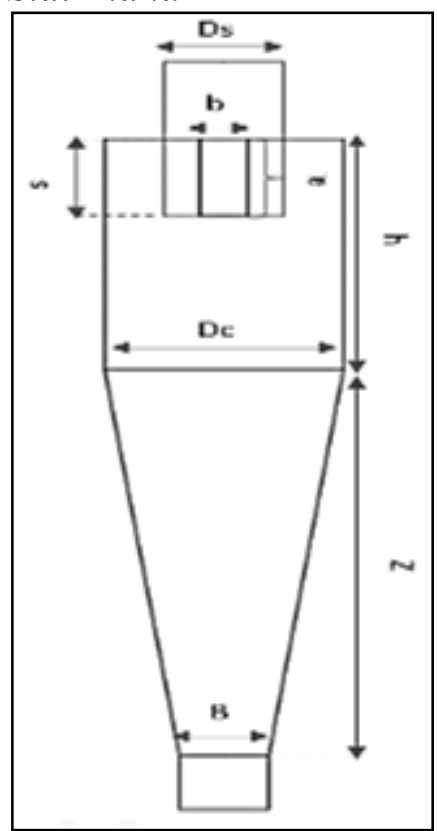

Nota. Representación dimensional de variables en ciclón, elaborado por el autor (2021).

\section{Ecuaciones para determinar el rendimiento del ciclón Stairmand}

Para el cálculo del rendimiento del ciclón se utilizaron las ecuaciones propuestas en la metodología empleada por (Tahir et al., 2020; Schnelle et al., 2016; Echeverri, 2006), cuyas variables se encuentran caracterizadas en tabla 1 . Es importante señalar que las variables implicadas son de entrada y salida, y están relacionadas con la velocidad equivalente, la velocidad de saturación, parámetros dimensionales del diseño del ciclón y la densidad del material, por mencionar algunas. Las ecuaciones son mostradas a continuación (Ecuaciones 9 a 13):

$$
\text { Veq }=\sqrt{\frac{4(\mathrm{Gr})\left(\mu_{\mathrm{A}}\right)\left(\rho_{\mathrm{p}}-\rho\right)}{3 \mathrm{p}^{3}}}
$$


Diseño y validación de un ciclón Stairmand destinado al filtrado parcial de material particulado

Vsat $=$

$\frac{4.913(\mathrm{Veq})\left(\mathrm{K}_{\mathrm{b}}^{0.4}\right)\left(\mathrm{D}_{\mathrm{C}}^{0.067}\right)\left(\sqrt[3]{\mathrm{Vi}^{2}}\right.}{\sqrt[3]{\mathrm{K}_{\mathrm{b}}}}$

$\mathrm{n}=1-(1-0.67(\mathrm{Dc} 0.14)$

$\left(\frac{\mathrm{T}}{283}\right) 0.3$

$\operatorname{Tr}=\frac{\left(\rho_{\mathrm{P}}\right)\left(\mathrm{Dpi}^{2}\right)}{18 \mu_{\mathrm{A}}}$

$\mathrm{ni}=1-\mathrm{e}^{\left(2\left(\frac{\mathrm{Gr} * \mathrm{Tr} * \mathrm{Q}(\mathrm{n}+1)}{\mathrm{Dc}^{3}}\right)(0.5 /(\mathrm{n}+1))\right)}$

\section{Simulación CFD}

Solidworks ${ }^{\circledR}$ Flow Simulation es una herramienta de análisis de fluidos como complemento del software Solidworks ${ }^{\circledR}$, permitiendo el análisis y solución de problemas relacionados a fluidos, a partir de las ecuaciones de Navier-Stokes. Para el análisis de CFD utilizando Solidworks ${ }^{\circledR}$ Flow Simulation, se emplea el desarrollo metodológico de (Matsson, 2018), resumido en el diagrama de Figura 2.

\section{Figura 2}

Metodología para simulación CFD en Solidworks ${ }^{\circledR}$ Flow Simulation

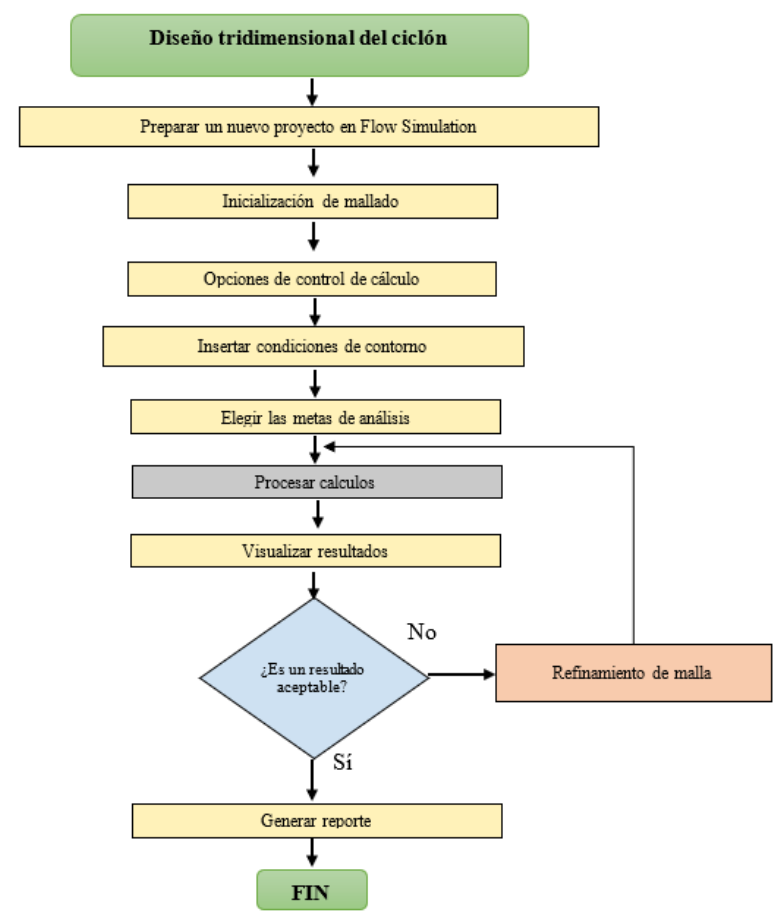

Nota. Resumen metodológico para simulación CFD, elaborado por Matsson (2018).

\section{Resultados}

Para determinar los parámetros geométricos a partir de las ecuaciones 1 a 8 , se establecieron las condiciones iniciales con base a la problemática planteada, las cuales se recopilan en Tabla 3, donde se especifican las condiciones cuantitativas iniciales del sistema. Aplicando las ecuaciones 1 a 9, se obtuvieron los parámetros geométricos del ciclón Stairmand, mostrados en tabla 4.

\section{Tabla 3}

Condiciones iniciales del sistema

\begin{tabular}{|c|c|}
\hline Variable & Valor \\
\hline $\begin{array}{l}\text { Densidad de la partícula } \\
\left(\mathrm{Kg} / \mathrm{m}^{3}\right)\end{array}$ & 1500 \\
\hline Temperatura de aire $\left({ }^{\circ} \mathrm{C}\right):$ & 450 \\
\hline Caudal de corriente $\left(\mathrm{m}^{3} / \mathrm{s}\right)$ & 3.2 \\
\hline Presión (kPa): & 85.3 \\
\hline $\begin{array}{l}\text { Concentración de partícula } \\
\left(\mathrm{g} / \mathrm{m}^{3}\right)\end{array}$ & 2 \\
\hline Velocidad inicial (m/s) & 22 \\
\hline $\begin{array}{l}\text { Viscosidad dinámica del aire } \\
(\mathrm{kg} / \mathrm{ms})\end{array}$ & $3.57 \times 10^{-5}$ \\
\hline Material particulado $\left(\mathrm{kg} / \mathrm{m}^{3}\right)$ & 0.411 \\
\hline $\begin{array}{l}\text { Área del conducto de entrada } \\
\left(\mathrm{m}^{2}\right)\end{array}$ & 0.1455 \\
\hline Velocidad equivalente $(\mathrm{W})$ & 1.6064 \\
\hline
\end{tabular}

Nota. Especificación cuantitativa de las condiciones iniciales del ciclón, elaborado por el autor (2021).

\section{Tabla 4}

Valores de los parámetros geométricos

Parámetro geométrico Valor en metros

\begin{tabular}{ll}
\hline $\mathbf{D c}$ & 1.35 \\
\hline $\mathbf{a}$ & 0.675 \\
\hline $\mathbf{b}$ & 0.27 \\
\hline $\mathbf{S}$ & 0.675 \\
\hline $\mathbf{D s}$ & 0.675 \\
\hline $\mathbf{h}$ & 2.025 \\
\hline $\mathbf{H}$ & 5.4 \\
\hline $\mathbf{z}$ & 3.375 \\
\hline $\mathbf{B}$ & 0.5063 \\
\hline Nota. Cuantificación de & parámetros geométricos,
\end{tabular}

elaborado por el autor (2021).

Con los valores de Tabla 4, se procedió a realizar el diseño en el software Solidworks ${ }^{\circledR}$, dando como resultado el modelo mostrado en Figura 3. Los diámetros aerodinámicos de las partículas presentes en la problemática, el porcentaje en masa, el tiempo de retención y el porcentaje de eficiencia de colección, se pueden 
observar en Tabla 5, a partir de las ecuaciones 9 a 13. Bajo las condiciones iniciales, se realizó el análisis de simulación con Solidworks ${ }^{\circledR}$ Flow Simulation para validar los resultados teóricos obtenidos en tabla 5. Los resultados obtenidos se muestran en figura 4.

\section{Figura 3}

Modelo tridimensional de ciclón Stairmand, diseñado con los parámetros teóricos calculados

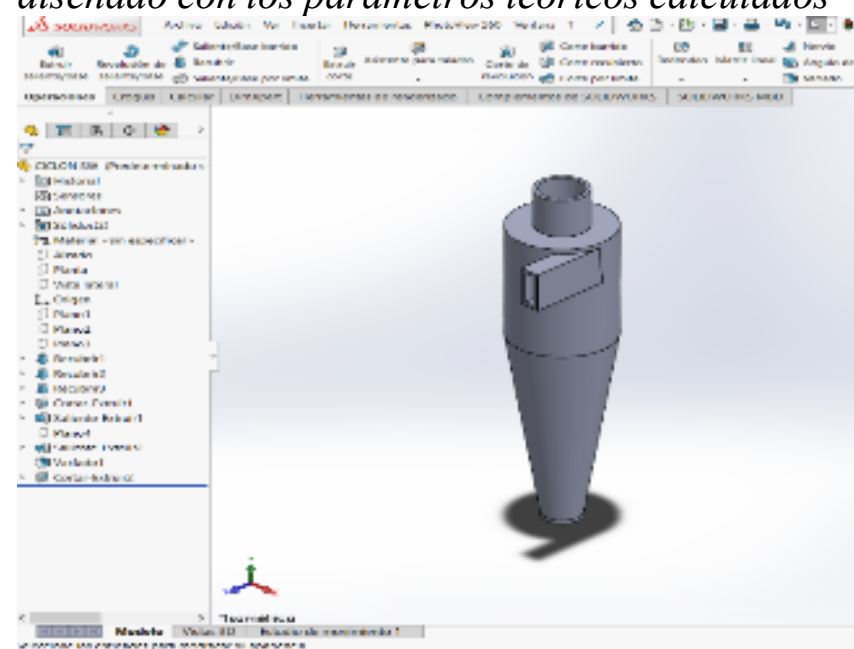

Nota. Modelos del ciclón en Solidworks ${ }^{\circledR}$, elaborado por el autor (2021).

\section{Tabla 5}

\begin{tabular}{lllllll}
\multicolumn{7}{l}{ Eficiencia total de colección } \\
\hline Tamaño & $\%$ & $\mathrm{Dp}$ & $\mathrm{Dp}$ & $\operatorname{Tr}(\mathrm{s})$ & $\mathrm{ni}$ & $\mathrm{ni}$ \\
aerodiná & en & $(\mu$ & $(\mathrm{m})$ & & & $\%$ \\
mico & $\mathrm{ma}$ & $\mathrm{m})$ & & & & $\mathrm{en}$ \\
$(\mu \mathrm{m})$ & $\mathrm{sa}$ & & & & & $\mathrm{mas}$ \\
& & & & & & $\mathrm{a}$ \\
\hline $\mathbf{5 - 2 0}$ & 100 & 12. & $12.5 \mathrm{x}$ & $3.668 \mathrm{x}$ & 0.7 & 79. \\
& & 5 & $10^{-6}$ & $10^{-4}$ & 94 & 40 \\
\hline
\end{tabular}

Nota. Porcentaje de colección para partículas de 5 a $20 \mu \mathrm{m}$, elaborado por el autor (2021).

\section{Figura 4}

Simulación CFD de eficiencia de colección en un rango de 10,000 partículas $(100 \%)$

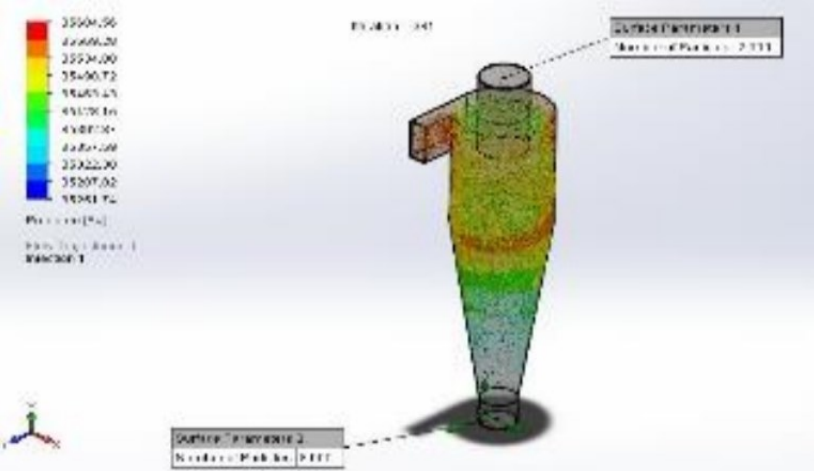

Nota. Análisis de simulación para eficiencia de colección, elaborado por el autor (2021).

\section{Conclusiones}

Se dimensionó un ciclón de alta eficiencia mediante las ecuaciones propuestas por Maduabuchi \& Kuye, 2017; Miller, 2017; Echeverri, 2006), para las condiciones de un proceso de corriente gaseosa propuesto por (Echeverri, 2006), teniendo como punto de partida la densidad de las partículas y del gas a una temperatura dada, además del caudal de entrada, presión de operación y concentración del PM.

Los datos del dimensionamiento generaron un modelo tridimensional en Solidworks ${ }^{\circledR}$ del ciclón de alta eficiencia tipo Stairmand. Posteriormente se procedió a realizar los cálculos para determinar de forma teórica el porcentaje de eficiencia del ciclón con base a las ecuaciones propuestas por (Tahir et al., 2020; Schnelle et al., 2016; Echeverri, 2006), haciendo uso de los parámetros geométricos calculados, además de las condiciones iniciales de operación del sistema, obteniendo un valor teórico de colección de $79.40 \%$ en partículas de 5 a $20 \mu \mathrm{m}$ (diámetro aerodinámico promedio de partícula equivalente a $12.5 \mu \mathrm{m}$ ), como se mostró en tabla 5 .

Con el modelo tridimensional del ciclón de alta eficiencia tipo Stairmand se procedió a realizar el análisis de simulación en CFD, agregando las mismas condiciones del sistema calculado de forma teórica, con la intención de validar el porcentaje de eficiencia de colección, en donde se estableció un total de 10 mil partículas entrantes al ciclón (este valor se tomó como $100 \%$ ), con un tiempo de retención simulado de 3.668 $\times 10-4$ segundos (iteración equivalente al valor teórico). En este tiempo, se colectaron 8 mil partículas, liberándose 2 mil al exteriro, equivalentes a un 80 y $20 \%$ respectivamente.

Estos datos demostraron que para las configuraciones geométricas y condiciones iniciales del problema de estudio, existe una discrepancia mínima (menor a 1\%) entre el cálculo teórico versus resultados de simulación en CFD con Solidworks ${ }^{\circledR}$ Flow Simulation, corroborando la hipótesis planteada en esta investigación, para el caso de estudio analizado. Los cálculos con CFD para el estudio de partículas con Solidworks ${ }^{\circledR}$ Flow Simulation son una herramienta invaluable en el diseño de 
dispositivos de separadores (Kurtin, 2020), ya que presentan gran precisión en los resultados obtenidos bajo los términos de los modelos teóricos (Alahmer \& Al-Dabbas, 2014; Gheorghe et al., 2018; Gopalakrishnan \& ArulPrakash, 2019).

La importancia de los análisis con CFD en el diseño y validación de ciclones, posibilita ajustar de forma rápida los modelos tridimensionales, permitiendo predecir casi de forma automática el comportamiento de los fluidos dentro del ciclón, e igualmente su eficiencia de colección. En este caso, los ajustes de diseño dentro de Solidworks ${ }^{\circledR}$, permiten generar nuevos resultados de simulación a partir de las condiciones de entrada y salida, únicamente modificando las condiciones geométricas.

El comportamiento de los flujos es competencia de la mecánica de fluidos, analizada desde diferentes perspectivas mediante pruebas experimentales con plantas piloto a través del funcionamiento de bombas hidráulicas (Barrios, Ferrer, \& Rosillón, 2020), o a partir del estudio de fenómenos físicos naturales o de laboratorio (Cengel \& Cimbala, 2018), es por ello, que se recomienda en futuras investigaciones, realizar pruebas experimentales de validación de los sistemas teóricos y simulados mediante CFD.

\section{Reconocimiento}

Agradezco al Dr. Pedro Jancarlo GómezVega quien funge como coautor de esta investigación, además por su apoyo como director de mi tesis doctoral, orientada al tema abordado en este trabajo.

\section{Referencias}

Alahmer, A., \& Al-Dabbas, M. (2014). Modeling and simulation study to predict the cement portland cyclone separator performance. Emirates Journal for Engineering Research, 19(1), 19-25.

An, Z., Jin, Y., Li, J., Li, W., \& Wu, W. (2018). Impact of Particulate Air Pollution on Cardiovascular Health. Current Allergy and Asthma Reports, 18(3). https://doi.org/10.1007/s11882-018-0768-8

Ast Ingeniería. (2016). Simulación de sistemas fluidodinámicos mediante la aplicación del método de los volúmenes finitos. http://www.ast-ingenieria.com/capacidadessoluciones/simulacion-cfd.
Bahamón, D., Alzate, H., \& Quintana, G. (2009). Simulación del patrón de flujo en fase simple para diferentes diseños de separadores ciclónicos. Revista Investigaciones Aplicadas, 6(1), 11-20.

Barrios, J., Ferrer, C., \& Rosillón, K. (2020). Planta piloto de bombas hidráulicas para la enseñanza y aprendizaje de la mecánica de fluidos. Revista TecnológicaEducativa Docentes 2.0, 9(1), 124-131. https://doi.org/10.37843/rted.v9i1.116

Cao, X., \& Bian, J. (2019). Supersonic separation technology for natural gas processing: A review. Chemical Engineering and Processing - Process Intensification, 136, 138-151. https://doi.org/10.1016/j.cep.2019.01.007

Capote, J., Alvear, A., Abreu, O., Lázaro, M., \& Espina, P. (2008). Influencia del modelo de turbulencia y del refinamiento de la discretizacion espacial en la exactitud de las simulaciones computacionales de incendios. Rev. Int. Met. Núm. Calc. Dis. Ing. 24,227-245.

Cengel, Y., \& Cimbala, J. (2018). Mecánica de fluidos: fundamentos $y$ aplicaciones (4a ed.). McGrawHill/Interamericana Editores, S.A. de C.V.

Echeverri, C. (2006). Diseño óptimo de ciclones. Revista Ingenierias Universidad De Medellín, 5(9), 123-139.

Echeverri, C. (2019). Contaminación atmosférica (20a ed., p. 86). Ediciones de la U.

EPA (2020). Particulate Matter (PM) Basics. US EPA. https://www.epa.gov/pm-pollution/particulate-matterpm-basics.

EPA-CICA (2012).Hoja de Datos. EPA. https://www3.epa.gov/ttncatc1/dir1/fcyclons.pdf

Eslava, J. (2016). Reflexiones acerca de la relación ambiente y saludables (1a ed., p. 103). Universidad Nacional de Colombia.

Fiordelisi, A., Piscitelli, P., Trimarco, B., Coscioni, E., Iaccarino, G., \& Sorriento, D. (2017). The mechanisms of air pollution and particulate matter in cardiovascular diseases. Heart Failure Reviews, 22(3), 337-347. https://doi.org/10.1007/s10741-017-9606-7

Gamiño, M., Castillo, F., Vázquez, R., Díaz, C., Guzmán, A., \& Herrera, F. (2018). Análisis del efecto geométrico de ciclones en el secado por aspersión de leche usando $C F D$. Avances en Ciencias e Ingeniería, 9, 11- 23.

Gheorghe, G., Mateescu, M., Persu, C., \& Gageanu, I. (2018). Theoretical simulation of air circulation inside cyclone mounted at exhaust outlet of pneumatic seed drill to optimize it. Engineering for Rural Development, 813817. https://doi.org/10.22616/erdev2018.17.n047

Gopalakrishnan, B., \& Arul-Prakash, K. (2019). Numerical study on pressure drops and filtration efficiency of gas-solid flow through axial cyclone separators. International Journal of Advances in Engineering Sciences and Applied Mathematics, 11(4), 280-287. 
Hadei, M., \& Naddafi, K. (2020). Cardiovascular effects of airborne particulate matter: A review of rodent model studies. Chemosphere, 242 , 125204 https://doi.org/10.1016/j.chemosphere.2019.125204

Hernández-Sampieri, R., \& Mendoza-Torres, C (2014). Metodología de la investigación (6th ed., p. 89). McGraw-Hill / Interamericana Editores, S.A. DE C.V.

Hoffmann, A., Stein, L., \& Bradshaw, P. (2008). Gas Cyclones and Swirl Tubes: Principles, Design and Operation. Applied Mechanics Reviews, 56(2), 28-29.

Khaniabadi, Y., Goudarzi, G., Daryanoosh, S., Borgini, A., Tittarelli, A., \& De Marco, A. (2016). Exposure to PM10, $\mathrm{NO} 2$, and $\mathrm{O} 3$ and impacts on human health. Environmental Science and Pollution Research, 24(3), https://doi.org/10.1007/s11356-016-8038-6.

Kirrane, E., Luben, T., Benson, A., Owens, E., Sacks, J., \& Dutton, S. (2019). A systematic review of cardiovascular responses associated with ambient black carbon and fine particulate matter. Environment International, 127, 305316. https://doi.org/10.1016/j.envint.2019.02.0277.

Kumar, V., \& Jha, K. (2018). Numerical investigations of the cone-shaped vortex finders on the performance of cyclone separators. Journal of Mechanical Science and Technology, 32(11), https://doi.org/10.1007/s12206-018-1028-5

Kurtin, K. (2020). Particle Separation using SOLIDWORKS Flow Simulation - Computer Aided Technology. Cati.com. Revisado el 28 octubre 2020, en https://www.cati.com/blog/2020/04/particle-separationusing-solidworks-flow-simulation/.

Kwiatkowski, S., Polat, M., Yu, W., \& Johnson, M. (2019). Industrial Emissions Control Technologies: Introduction. Encyclopedia of Sustainability Science and Technology, 1-35. https://doi.org/10.1007/978-1-49392493-6 1083-1

Kyung, S., \& Jeong, S. (2020). Particulate-Matter Related Respiratory Diseases. Tuberculosis and Respiratory Diseases, 83(2),

https://doi.org/10.4046/trd.2019.0025

Leikauf, G., Kim, S., \& Jang, A. (2020). Mechanisms of ultrafine particle-induced respiratory health effects. Experimental \& Molecular Medicine, 52(3), 329-337. https://doi.org/10.1038/s12276-020-0394-0.

Lucas, D. (2016). Qualification of CFD-models for multiphase flows. Kerntechnik, 81(2), 167-169. https://doi.org/10.3139/124.110686

Luciano, R., Silva, B., Rosa, L., \& Meier, H. (2018). Multiobjective optimization of cyclone separators in series based on computational fluid dynamics. Powder Technology, 325, pp.452-466.

Maduabuchi, F., \& Kuye, A. (2017). Optimization of Cyclone Design Parameters using Java in Netbeans Integrated Development Environment (IDE) Programme. Journal of Scientific and Engineering Research, 4(8), 221-230.
Makwana, P., \& Lakdawala, A. (2016). CFD Analysis of Particle Laden Flow in a Cyclone Separator Using RANS and LES Methodologies. Fluid Mechanics and Fluid PowerContemporary Research, 669-678. https://doi.org/10.1007/978-81-322-2743-4_64

Mannucci, P. (2017). Air pollution levels and cardiovascular health: Low is not enough. European Journal of Preventive Cardiology, 24(17), 1851-1853. https://doi.org/10.1177/2047487317719356

Matsson, J. (2018). An Introduction to SOLIDWORKS Flow Simulation 2018 (1a ed., p. 1). SDC Publications.

Miller, B. (2017). Introduction to Coal Utilization Technologies (2a ed., pp. 147-229). Elsevier.

Petit, A., Pico, H., \& Barbosa, M. (2012). Influence of the cyclone cone length on flow variables. Avances En Ciencias E Ingeniería, 3(3), 103-118.

Petit, A., \& Barbosa, M. (2013). Simulación de flujo turbulento dentro de un separador tipo ciclón usando Large Eddy Simulation. Asociación Argentina De Mecánica Computacional, 1(1)

Schnelle, K., Dunn, R., \& Ternes, M. (2016). Air pollution control technology handbook (2a ed., pp. 334-335). Taylor \& Francis.

Sicard, P., Khaniabadi, Y., Perez, S., Gualtieri, M., \& De Marco, A. (2019). Effect of O3, PM10 and PM2.5 on cardiovascular and respiratory diseases in cities of France, Iran, and Italy. Environmental Science and Pollution Research, 26(31), 32645-32665. https://doi.org/10.1007/s11356-019-06445-8

Sofia, D., Gioiella, F., Lotrecchiano, N., \& Giuliano, A. (2020). Mitigation strategies for reducing air pollution. Environmental Science and Pollution Research, 27(16), https://doi.org/10.1007/s11356-020-08647-x

Sotomayor, A. (2018). Tecnologías limpias (1a ed.). Universidad de Lima, Fondo Editorial.

Tahir, M., Mursalim, M., Salengke, S., \& Metusalach, M. (2020). Design and performance of a cyclone separator integrated with heat exchanger for smoked fish production. $A R P N$ Journal of Engineering and Applied Sciences, 12(19), 5398.

Vakamalla, T., Vadlakonda, B., Aketi, V., \& Mangadoddy, N. (2016). Multiphase CFD Modelling of Mineral Separators Performance: Validation Against Tomography Data. Transactions of The Indian Institute of Metals, 70(2), 323-340. https://doi.org/10.1007/s12666-016-0995-4.

Virto, L. (2017). Dinámica de gases (1a ed., p. 111). UPCGRAU.

Wang, S., Li, H., Wang, R., Wang, X., Tian, R., \& Sun, Q. (2019). Effect of the inlet angle on the performance of a cyclone separator using CFD-DEM. Advanced Powder Technology, 30(2), https://doi.org/10.1016/j.apt.2018.10.027

Wasilewski, M., \& Brar, L. (2019). Effect of the inlet duct angle on the performance of cyclone separators. Separation and 
Diseño y validación de un ciclón Stairmand destinado al
Purification
Technology, 213,
19-33.

https://doi.org/10.1016/j.seppur.2018.12.023

Witt, P., Mittoni, L., Wu, J., \& Shepherd, I. (1999). Validation of a CFD model for predicting gas flow in a cyclone. Proceedings of CHEMECA99. http://hdl.handle.net/102.100.100/215218?index=1.

World Health Organization. (2020). Technology and designbased interventions. https://www.who.int/airpollution/household/intervention s/technology/en/.

Xamán, J., \& Girón, M. (2015). Dinámica De Fluidos Computacional Para Ingenieros (1a ed.). Palibrio.

Yin, P., Guo, J., Wang, L., Fan, W., Lu, F., \& Guo, M. (2020). Higher Risk of Cardiovascular Disease Associated with Smaller Size-Fractioned Particulate Matter. Environmental Science \& Technology Letters, 7(2), 95-101. https://doi.org/10.1021/acs.estlett.9b00735

Yao, Z., You, S., Dai, Y., \& Wang, C. (2018). Particulate emission from the gasification and pyrolysis of biomass: Concentration, size distributions, respiratory depositionbased control measure evaluation. Environmental Pollution, 242, $1108-1118$. https://doi.org/10.1016/j.envpol.2018.07.126 